\title{
Numerical simulation of radiation in high altitude solid propellant rocket plumes
}

\author{
Quentin Binauld $^{a b}$, Jean-Michel Lamet ${ }^{a \star}$, Lionel Tessé ${ }^{a}$, Philippe Rivière ${ }^{b}$, \\ Anouar Soufiani ${ }^{b}$ \\ ${ }^{a}$ ONERA The French Aerospace Lab \\ F-31055, Toulouse, France \\ ${ }^{b}$ Laboratoire EM2C, CNRS, CentraleSupélec, Universite Paris Saclay, \\ 8-10 rue Joliot-Curie, 91190 Gif-sur-Yvette, France \\ *Corresponding author : Jean-Michel.Lamet@onera.fr
}

\begin{abstract}
Models and computational schemes are presented to simulate the flow field and radiation in solid propellant rocket plumes at high altitude. Different models are developed to calculate plume radiation induced by both the gas and the dispersed phase of alumina particles. Simulations were made to reproduce a $110 \mathrm{~km}$ altitude rocket plume and comparisons with experimental data on the plume radiance are presented. Influence of different physical processes on the simulation of the plume flow field and radiation is discussed, such as alumina phase change, scattering and the coupling of radiation with the two-phase flow.

Keywords: solid propellant rocket plumes, alumina particles, exhaust gases, CFD (Computational Fluid Dynamics), SNB (Statistical Narrow-Band) radiation models, Monte Carlo simulation.
\end{abstract}

\section{Introduction}

In the past decades, many studies have been carried out on solid propellant rocket plumes, however, most of them were focused on relatively low to medium altitudes [1]. This study deals with the simulation of high altitude plumes, above $100 \mathrm{~km}$, for which radiation may have an important role. Those plumes are characterized by a highly rarefied two-phase flow composed of alumina particles and exhaust gases produced by the combustion of a solid propellant in the combustion 
chamber. In these conditions, the gas may go through a high degree of thermal desequilibrium.

Radiation from solid fuel rocket plumes is significant factor to estimate heat fluxes on the walls of vehicles and to obtain accurate description of the condensed phase such as the phase state and temperature of alumina particles. This last point is in particular crucial for radiative signature prediction. Indeed, at the nozzle exit, the condensed phase which represents about $30 \%$ of the mass flow rate, is composed of liquid alumina particles whose diameters range from 0.1 to $10 \mu \mathrm{m}$. As the plume expands, alumina is cooled by convective and radiative transfer with the gas phase and starts to crystallize following a supercooling process. In the plume nearfield zone, the cooling of alumina particles is mainly driven by the convective transfer, due to the high velocity and temperature differences between both phases. Further downstream, the convective heat transfer is negligible as the pressure is very low (rarefied gas), and the radiative transfer determines the cooling of particles. Therefore, in order to simulate the flowfield, a suitable model is needed for the gas/particle interactions, the phase change of alumina and radiative properties of both phases.

Various simulations have been done on the radiation of high altitude plumes. Some of these simulations, as those of Candler et al. [2], Vitkin et al. [3], were carried out solving Navier-Stokes equations for the gas simulation. For those studies, the radiative calcutations have been carried out with crude modeling and was uncoupled with the plume flow field. Burt and Boyd [4] conducted their studies using a DSMC-based simulation (Direct Simulation Monte Carlo) which allows to treat rarefied aspects of the plume. Radiation calculation was coupled with the flow field but did not take into account gas radiation and simple models were used to calculate the absorption and scattering by the particles. The gas radiation may be an important issue for plume simulations, not only for signature predictions but also for gas/particle radiative interaction.

Our study deals with simulation of the "Bow Shock Ultraviolet 2" experiment [5] (BSUV2), which is, to our knowledge, the only experiment available which gives experimental data on radiation for high altitude solid propellant rocket plumes. In this experiment, spectrometers and photometers, fixed on the vehicle, were used to measure the radiation emitted in the UV range by the plume during the flight of both second and third stages of a rocket at altitudes between 100 and $115 \mathrm{~km}$. We present in this article results of simulation on the second stage, for which radiation measurement were carried out during the Antares II motor burn at an altitude of $109.6 \mathrm{~km}$. Simulations have been made using the multiphysics platform CEDRE [6] developed by ONERA which allows three-way coupling be- 
tween gas, particulate phase and radiation. The gas phase is resolved using a Navier-Stokes solver which does not take into account rarefied and desequilibrium aspects but is efficient enough to simulate the main core of the plume. The condensed phase is simulated with a solver based on an Eulerian approch. Details about the two-phase flow modeling with gas/particle interactions are given in section 2. Radiation is dealt with a radiative solver based on a Monte-Carlo algorithm. Radiative property models, in particular for gas species, and radiative transfer methods have been developed to simulate accurately the radiative transfer in the plume and the interactions between the two-phase flow and radiation. This point is described in section 3 . The last section is devoted to simulation results and comparisons with the experimental data.

\section{Two-phase flow modeling}

The plume is simulated as a two phase flow, a gas phase and a particle phase. Several approximations are made in order to simplify the study and decrease the computational cost of the simulation. The plume is assumed to be at steady state, axisymmetrical, non turbulent and non reactive as the gas mean free-path is low because of the low gas density. Inside the flow, alumina particles and the gas may have different velocities and temperature distributions. Moreover, as there are several sizes of particles, each size class is associated with its own thermophysical properties. For each phase, the solving of the flow field is determined by the mass, momentum and energy conservation equations. Momentum and energy exchanges between gas and particles induce source terms to the equations of each phase.

\subsection{Gas phase modeling}

The gas flow field is simulated using a code which solves the compressible Navier-Stokes equations for a multi-species gas using a finite-volume method. The Knudsen number which characterizes the flow regime, is sufficiently low in most of the flow field to enable the simulation of the main core of the plume with a continuum approach. In the region with relatively high Knudsen number, an alternative approach would be more appropriate to simulate the flow field taking into account rarefaction aspects. However, in the plume, these areas are located in the backstream region above the nozzle lip and in the diffuse shock wave between the atmosphere and exhaust gases. Even if the temperature is quite high in these regions, the gas is rarefied and there are nearly no particles, so that they would not 
contribute significantly to the heat fluxes at the body of the vehicle nor the global radiation in the flowfield.

The gas phase is composed of $N_{m}$ different species and the diffusion between the exhaust gases coming from the nozzle and the free-stream gases of the atmosphere is taken into account. The governing equations can be written as

$$
\frac{\partial \boldsymbol{Q}}{\partial t}+\nabla \cdot \boldsymbol{F}_{c}=\nabla \cdot \boldsymbol{F}_{\boldsymbol{d}}+\boldsymbol{S}_{g}
$$

where $\boldsymbol{Q}$ is the vector of conserved quantities, $\boldsymbol{F}_{\boldsymbol{c}}$ is the vector of convective fluxes, $\boldsymbol{F}_{\boldsymbol{d}}$ the vector of diffusive fluxes and $\boldsymbol{S}_{\mathrm{g}}$ the vector of source terms due to radiation and gas/particle interaction. Those vectors are given by

$$
\boldsymbol{Q}=\left(\begin{array}{c}
\rho Y_{1} \\
\mid \\
\rho Y_{N_{m}} \\
\rho \boldsymbol{u} \\
\rho e_{t}
\end{array}\right) \boldsymbol{F}_{\boldsymbol{c}}=\left(\begin{array}{c}
\rho Y_{1} \boldsymbol{u} \\
\mid \\
\rho Y_{N_{m}} \boldsymbol{u} \\
\rho \boldsymbol{u} \otimes \boldsymbol{u}+P \mathbf{I} \\
\rho\left(e_{t}+\frac{P}{\rho}\right) \boldsymbol{u}
\end{array}\right) \quad \boldsymbol{F}_{\boldsymbol{d}}=\left(\begin{array}{c}
-\boldsymbol{J}_{1} \\
\mid \\
-\boldsymbol{J}_{N_{m}} \\
\boldsymbol{\tau} \\
\boldsymbol{\tau} \cdot \boldsymbol{u}-\boldsymbol{J}_{e}
\end{array}\right) \quad \boldsymbol{S}_{g}=\left(\begin{array}{c}
0 \\
\mid \\
0 \\
\boldsymbol{S}_{d} \\
\boldsymbol{S}_{e}+P_{\mathrm{rad}}
\end{array}\right),
$$

where $\rho$ is the density of the gas, $Y_{i}$ is the mass fraction of molecular species $i, \boldsymbol{u}$ is the hydrodynamic velocity of the gas and $e_{t}$ is the specific total energy of the gas. Thermodynamic equations of state permit to pass from the conservative quantities to the natural quantities, the pressure $P$ and temperature $T$

$\rho e_{t}=\frac{1}{2} \rho\|\boldsymbol{u}\|^{2}+\sum_{i=1}^{N_{m}} \rho Y_{i} e_{i n t}^{i}(T), \quad P=\sum_{i=1}^{N_{m}} \rho Y_{i} \frac{R}{M_{i}}, \quad e_{i n t}^{i}(T)=e_{i n t}^{i}\left(T_{0}\right)+\int_{T_{0}}^{T} c_{\nu}^{i}(T) d T$,

where $R$ is the universal gas constant, $M_{i}$ the molar mass of species $i, e_{i n t}^{i}$ its internal ernergy and $c_{v}^{i}(T)$, its specific heat calculated as a polynomial function of the temperature. In the diffusive fluxes vector, the diffusion flux $\boldsymbol{J}_{i}$ is calculated according to the Fick's Law as

$$
J_{i}=-\rho D_{i} \nabla Y_{i},
$$

where $D_{i}$ is the diffusion coefficient of the species $i$ calculated with a Schmidt number equal to one. $\tau$ is the viscous stress tensor for which the dynamic viscosity of each species is calculated with the Sutherland law. $\boldsymbol{J}_{e}$ stands for the energy diffusion vector which includes the conductive heat flux calculated with the Fourier law, and the molecular diffusion fluxes as

$$
\boldsymbol{J}_{e}=-\lambda_{g} \nabla T+\sum_{i=1}^{N_{m}} h_{i} \boldsymbol{J}_{i}
$$


where $\lambda_{g}$ is the thermal conductivity of the fluid and $h_{i}$ the specific enthalpy of the species $i$. In the source term vector, $S_{d}$ and $S_{e}$ components are relative to momentum and convective exchanges between gas and particules, respectively. These terms are discussed in section 2.3. The term $P_{\text {rad }}$ is the radiative power only due to the gas and is detailed in section 3.3.

To solve these equations we used the finite volume method with a first order implicit scheme for the temporal integration, a second order scheme for space integration and an HLLC type scheme (Harten-Lax-van Leer Contact wave) [7] for the calculation of the convective fluxes between cells.

\subsection{Condensed phase modeling}

To simulate the particle phase we use an Eulerian finite volume solver. The condensed phase is modeled by $N_{c}$ classes of spherical particles, each class $k$ having its own characteristic diameter $D^{(k)}$, temperature $T_{p}^{(k)}$, a number density of particles $n^{(k)}$, a volume fraction $\alpha^{(k)}$, a mean velocity $\boldsymbol{v}^{(k)}$ and an specific internal energy $e_{i n t}^{(k)}$. As for the fluid, for each class, the evolution of those variables is ruled by their own conservation equations. Note that each class is inert toward the others, and only the gas phase and radiation influence its evolution.

The temperature non-uniformity during particle solidification was discussed in Refs. [8, 9] for relatively large particles. However, for the small alumina particles considered in this work, it can be easily shown that the conductive time scale of a given particle is small compared to convective and radiative time scales, and to the residence time of the particles in the plume. It is therefore reasonable to consider isothermal particles in the present application.

The system of governing equations for a particle class $k$ can be written as

$$
\frac{\partial \boldsymbol{Q}_{p}^{(k)}}{\partial t}+\nabla \cdot \boldsymbol{F}_{p}^{(k)}=\boldsymbol{S}_{p}^{(k)},
$$

where $\boldsymbol{Q}_{p}^{(k)}$ is the vector of conserved quantities, $\boldsymbol{F}_{p}^{(k)}$ the vector of convective fluxes and $\boldsymbol{S}_{p}^{(k)}$ the source term vector. These vectors take the form of

$$
\boldsymbol{Q}_{p}^{(k)}=\left(\begin{array}{c}
n^{(k)} \\
\alpha^{(k)} \rho_{A l} \\
\alpha^{(k)} \rho_{A l} \boldsymbol{v}^{(k)} \\
\alpha^{(k)} \rho_{A l} e_{i n t}^{(k)}
\end{array}\right) \quad \boldsymbol{F}_{p}^{(k)}=\left(\begin{array}{c}
n^{(k)} \boldsymbol{v}^{(k)} \\
\alpha^{(k)} \rho_{A l} \boldsymbol{v}^{(k)} \\
\alpha^{(k)} \rho_{A l} \boldsymbol{v}^{(k)} \otimes \boldsymbol{v}^{(k)} \\
\alpha^{(k)} \rho_{A l} e_{i n t}^{(k)} \boldsymbol{v}^{(k)}
\end{array}\right) \quad \boldsymbol{S}_{p}^{(k)}=\left(\begin{array}{c}
0 \\
0 \\
n^{(k)} \boldsymbol{F}_{d r}^{(k)} \\
n^{(k)} \phi_{c}^{(k)}+P_{\text {rad }}^{(k)}
\end{array}\right) .
$$

Here, $\rho_{A l}$ is the density of alumina (assumed a constant equal to $2700 \mathrm{~kg} \cdot \mathrm{m}^{-3}$ ), $\boldsymbol{F}_{d r}^{(k)}$ and $\phi_{c}^{(k)}$ are respectively the drag force and the convective heat flux caused 
by the gas on a particle, and $P_{\text {rad }}^{(k)}$ the radiative power only due to the class $k$ (see section 3.3).

The alumina particles solidify following a supercooling process, that is to say, during its cooling along the plume, they remain in liquid state above the temperature of nucleation $T_{c}$ of $1930 \mathrm{~K}$. When a particle reaches this temperature it starts to solidify at a constant temperature $T_{m}$ of $2289 \mathrm{~K}$, the melting temperature of alumina. Once totally solidified, the particle temperature decreases again due to radiative and convective cooling. A modeling of this phenomenon has been developed and allows us to express the internal energy $\mathrm{e}_{i n t}^{(k)}$ of a class $k$ as a bijective function of its temperature $T_{p}^{(k)}$ and its mass fraction of solid state phase $\chi^{(k)}$.

$$
\left\{\begin{array}{c}
e_{i n t}^{(k)}\left(\chi^{(k)}, T_{p}^{(k)}\right)=\chi^{(k)} c_{p, s o l}\left(T_{p}^{(k)}\right) T_{p}^{(k)}+\left(1-\chi^{(k)}\right)\left[L_{f u s}+c_{p, l i q}\left(T_{p}^{(k)}\right) T_{p}^{(k)}\right] \\
e_{s o l}=c_{p, s o l}\left(T_{m}\right) T_{m} \\
e_{l i q}=c_{p, l i q}\left(T_{c}\right) T_{c}+L_{f u s}
\end{array}\right.
$$

where $L_{f u s}$ is the latent heat of fusion equal to $1.0710^{6} \mathrm{~J} / \mathrm{kg}, c_{p, l i q}$ is the specific heat of liquid alumina (assumed a constant equal to $1850 \mathrm{~J} / \mathrm{kg} / \mathrm{K}$ ) and $c_{p \text {,sol }}$ the specific heat of solid alumina that can be expressed as a polynomial function of the temperature. With this modeling, the particle is totally liquid if $e_{i n t}$ is above $e_{\text {liq }}$, and totally solid when $e_{i n t}$ is below $e_{\text {sol }}$. When a liquid particle reaches $T_{c}$, it undergoes a spontaneous partial crystallization and its temperature instantaneously changes to the melting temperature $T_{m}$ while its internal energy remains conserved. In the plume, the heat created during the solidification of the alumina compensates with the cooling caused by interaction with the gas phase and by radiation. The phase change of alumina plays an important role not only to establish the correct temperatures of alumina in the plume but also because the radiative properties of alumina are different depending on its phase state. Moreover, the supercooling phenomenon tends to decrease the convective heat transfer between gas and particles, as the liquid particles are colder than the melting temperature and so slows down the cooling of alumina particles.

To solve the system of conservation equations for the condensed phase, we used a second order explicit scheme for the temporal integration, a second order spatial scheme and a Godunov [10] type scheme for the calculation of the fluxes at the faces of the mesh cells.

\subsection{Gas/particle interactions}

The momentum and energy exchanges between the gas phase and the particles are important for an accurate simulation of the flowfield as they determine the 
cooling of alumina particles during the expansion of the plume. Because of the rarefied flow field, adequate modeling needs to be employed to evaluate momentum and energy exchange between both phases. In this work, we use modeling suitable for highly compressible flows.

The drag force $\boldsymbol{F}_{d r}^{(k)}$ applied by the gas phase on a particle is responsible for momentum exchange between both phases, it is expressed as

$$
\boldsymbol{F}_{d r}^{(k)}=\frac{1}{8} \pi\left(D^{(k)}\right)^{2} \rho C_{D}\left\|\boldsymbol{u}-\boldsymbol{v}^{(k)}\right\|(\boldsymbol{u}-\boldsymbol{v}),
$$

where $C_{D}$ is the drag coefficient whose expression depends on the particle Reynolds number $\operatorname{Re}_{p}$ based on the relative velocity and the dynamic viscosity $\mu_{g}$ of the gas by

$$
\operatorname{Re}_{p}=\frac{\rho D^{(k)}\left\|\boldsymbol{u}-\boldsymbol{v}^{(k)}\right\|}{\mu_{g}} .
$$

For low Reynolds number as in plume flow field, Carlson and Hoglund [11] proposed the following expression of the drag coefficient :

$$
C_{d}=\frac{24}{\operatorname{Re}_{p}} \frac{\left(1+0.15 \operatorname{Re}_{p}^{0.687}\right)\left[1+\exp \left(-\frac{0.427}{\mathrm{M}_{p}^{4.63}}-\frac{3}{\operatorname{Re}_{p}{ }^{0.88}}\right)\right]}{1+\frac{\mathrm{M}_{p}}{\operatorname{Re}_{p}}\left[3.82+1.28 \exp \left(-1.25 \frac{\mathrm{Re}_{p}}{\mathrm{M}_{p}}\right)\right]},
$$

where $\mathrm{M}_{p}$ is the particle Mach Number based on the relative velocity of both phases.

For highly compressible flows, Carlson and Hoglund [11] also developed in their work an expression of the convective heat transfer $\phi_{c}^{(k)}$ given by the gas to a particle

$$
\begin{gathered}
\phi_{c}^{(k)}=\pi D^{(k)} \mathrm{Nu} \lambda_{g}\left(T-T_{p}^{(k)}\right), \\
\mathrm{Nu}=\frac{2+0.6 \operatorname{Re}_{p}^{\frac{1}{2}} \operatorname{Pr}^{\frac{1}{3}}}{\left[1+3.42 \frac{\mathrm{M}_{p}}{\operatorname{Re}_{p} \operatorname{Pr}}\left(2+0.6 \operatorname{Re}_{p} p^{\frac{1}{2}} \operatorname{Pr}^{\frac{1}{3}}\right)\right]},
\end{gathered}
$$

where $\mathrm{Nu}$ is the Nusselt number expressed as a function of $\operatorname{Re}_{p}, \mathrm{M}_{p}$ and $\operatorname{Pr}$ the Prandt number of the gas phase.

In Eq. (2), the terms $\boldsymbol{S}_{d}$ and $\boldsymbol{S}_{e}$ are also related to $\boldsymbol{F}_{d r}^{(k)}$ and $\phi_{c}^{(k)}$ through the following expressions

$$
\boldsymbol{S}_{d}=-\sum_{k=1}^{N_{c}} n^{(k)} \boldsymbol{F}_{d r}^{(k)}, \quad \boldsymbol{S}_{e}=-\sum_{k=1}^{N_{c}} n^{(k)} \phi_{c}^{(k)},
$$

For the strong gas/particle coupling, we use the Lie splitting method [12] which consists of temporally integrating the source terms after a first calculation of transport phenomena. 


\section{Radiation Modeling}

The simulation of the two phase flow field informs about temperature, pressure and density fields, necessary to calculate the radiation field. For radiative calculation in high altitude plumes, accurate modeling of radiative properties for both gas and particles, as well as suitable methods to solve the radiative transfer equation, are required. The plume radiation is calculated from infrared to UV range, for wavelengths from 0.2 to $200 \mu \mathrm{m}$, taking into account the scattering by the particles.

\subsection{Radiative properties of alumina particles}

Absorption and scattering coefficients, and the phase function of alumina particles, depend on the size of particles and the complex refractive index of alumina, $m=n-i k$. Considering that the size of particules in our application is about a few micrometers, Mie theory [13] is used to calculate these radiative properties. Many investigations have been made in the past decades in order to find expressions of the refractive index $n$ and the absorption index $k$ as functions of the temperature and the wavelength $[14,15]$. Strong discrepancies exist between those studies as the results for $k$ may vary by several orders of magnitude depending on the temperature and wavelength range of the studies and the type of investigated alumina (liquid phase or crystalline phase of solid alumina). For our study we use the expression given by Dombrovsky [16] for the refractive index $n$,

$$
n=\left[1+\lambda^{2}\left(\frac{1.024}{\lambda^{2}-0.00376}+\frac{1.058}{\lambda^{2}-0.01225}+\frac{5.281}{\lambda^{2}-321.4}\right)\right]^{0.5}\left(0.9904+2.02 \times 10^{-5} T_{p}\right),
$$

where $\lambda$ is the wavelength in $\mu \mathrm{m}$, and $T_{p}$ the particle temperature in Kelvin.

The absorption index of alumina strongly depends on its phase state. For our study, we do not take into account the different crystalline phases of solid alumina. We choose to employ two different models for the expression of the absorption index of alumina in solid or liquid state. For liquid alumina we use the empirical expression developed by Dombrovsky [16]

$$
k_{\text {liq }}=0.002\left(0.06 \lambda^{2}+0.7 \lambda+1\right) \exp \left[1.847\left(\frac{T_{p}}{1000}-2.95\right)\right] .
$$

For the solid alumina, we employ the semi-empirical model of Anfimov [17] based on the different mechanisms responsible for the absorption of radiation, the absorption by the free electrons, by the crystal lattice, by the valence electrons and the Urbach edge absorption. 
Using the Mie theory, the absorption coefficient, the scattering coefficient and the phase function are calculated using a Gaussian distribution around the mean radius $r^{(k)}$ of each class of particles with a standard deviation of $r^{(k)} / 10$. In the case of alumina particles in both liquid and solid states, the radiative properties are calculated as if the particles were divided into two groups of completely solid and completely liquid particles, respecting the mass fraction ratio.

\subsection{Gas radiation models}

From the exhaust gases leaving the nozzle, $\mathrm{CO}, \mathrm{CO}_{2}, \mathrm{HCl}$ and $\mathrm{H}_{2} \mathrm{O}$ are the ones that contribute the most to the radiation. Radiative properties of the gases originate from several million transition lines whose profiles are determined by Doppler and collisional (Lorentz) broadening. To model the radiative properties of gases and solve the radiative transfer equation (RTE), the line-by-line approach, which gives absorption coefficients with a spectral resolution sufficient to describe the line profile (typically $10^{-3} \mathrm{~cm}^{-1}$ for our applications), is considered as a reference. However, for engineering applications, using line-by-line models has a considerable computational cost. Therefore, it is necessary to employ models of radiative properties averaged on wider spectral bands. For the study of plume radiation at high altitude, we choose to use statistical narrow-band models (SNB), for which the absorption spectrum is divided into bands of $25 \mathrm{~cm}^{-1}$. These models are adequate for our study as they allow us to describe the radiative properties of gases over a wide range of temperatures and pressures and permit the solution of the RTE for strongly non-uniform media.

For a uniform column of length $l$, the SNB model of Mayer and Goody [18] leads to the average transmissivity over a range of wave number $\sigma$ of width $\Delta \sigma$ as

$$
\bar{\tau}=\frac{1}{\Delta \sigma} \int_{\Delta \sigma} \exp \left(-\kappa_{\sigma} l\right) d \sigma=\exp \left(-\frac{\bar{W}}{\delta}\right) .
$$

Here $\delta$ is the mean spacing between lines inside $\Delta \sigma$ and $\bar{W}$ is the mean value of the equivalent black line width. Various models of $\frac{\bar{W}}{\delta}$ have been developed, depending on the broadening regime of the line and various line intensity probability distributions.

For Lorentzian broadening regime, important at relatively high pressures, the exponential tail inversed distribution [19] was shown to accurately model curves of growth of $\frac{\overline{W_{L}}}{\delta}$. The mean equivalent black width is then expressed as

$$
\frac{\bar{W}_{L}}{\delta}=\frac{\bar{\beta}_{L}}{\pi}\left(\sqrt{1+\frac{2 \pi \bar{k}_{L} p_{e} l}{\bar{\beta}_{L}}}-1\right) .
$$


The parameter $\bar{k}_{L}$ stands for the mean absorption coefficient per partial pressure $p_{e}$ of the gas species $e$, in $\left(\mathrm{m}^{-1} \mathrm{~Pa}^{-1}\right)$ and $\bar{\beta}_{L}$ is a parameter that characterizes how the lines overlap on the spectral band, and varies linearly with the total pressure. In the same way, at low pressure, the Doppler broadening regime dominates, and the mean equivalent black line width can be expressed with parameters $\bar{k}_{D}$ and $\bar{\beta}_{D}$ according to generalized Malkmus distribution [20, 21]

$$
\frac{\bar{W}_{D}}{\delta}=\bar{\beta}_{D} H_{\alpha}\left(\frac{\bar{k}_{D} p_{e} l}{\bar{\beta}_{D}}\right), \quad H_{\alpha}(x)=\frac{1}{\alpha \sqrt{\pi}} \int_{-\infty}^{+\infty}\left[\left(1+x \exp \left(-\xi^{2}\right)\right)^{\alpha}-1\right] d \xi,
$$

where $\alpha$ has different values depending on the species (Table 1) and was chosen to provide the best fit of the curves of growth.

For our study, considering that the pressure varies from a few $\mathrm{Pa}$ to a few bars, we use the Voigt regime for which the mean black equivalent line width, $\bar{W}_{V}$, can be expressed as a function of $\bar{W}_{D}$ and $\bar{W}_{L}$ thanks to the Ludwig formula [22]

$$
\begin{gathered}
\frac{\bar{W}_{V}}{\delta}=p_{e} l \bar{k} \sqrt{1-\Omega^{-\frac{1}{2}}} \\
\Omega=\left[1-\left(\frac{1}{p_{e} l \bar{k}_{L}} \frac{\bar{W}_{L}}{\delta}\right)^{2}\right]^{-2}+\left[1-\left(\frac{1}{p_{e} l \bar{k}_{D}} \frac{\bar{W}_{D}}{\delta}\right)^{2}\right]^{-2}-1 .
\end{gathered}
$$

The SNB model parameters $\bar{k}_{D}, \bar{k}_{L}, \bar{\beta}_{D}$ and $\bar{\beta}_{L}$ have been calculated and tabulated with the temperature, from 100 to $5000 \mathrm{~K}$, for the molecules $\mathrm{H}_{2} \mathrm{O}, \mathrm{CO}_{2}, \mathrm{HCl}$ and $\mathrm{CO}$, for a spectral range between 50 and $11250 \mathrm{~cm}^{-1}$ and a band width of $25 \mathrm{~cm}^{-1}$ using high resolution spectroscopic databases (Table 1). Lorentzian parameters have been calculated at a total pressure of 1 bar.

Table 1: Number of bands, minimum and maximum wave number, spectroscopic database, and parameter $\alpha$ used for the SNB models

\begin{tabular}{|c|c|c|c|c|c|}
\hline Molecule & Number of bandes & $v_{\min }\left(\mathrm{cm}^{-1}\right)$ & $v_{\max }\left(\mathrm{cm}^{-1}\right)$ & Spectroscopic database & $\alpha$ \\
\hline $\mathrm{H}_{2} \mathrm{O}$ & 449 & 50 & 11250 & HITEMP 2010 [23] & 0.15 \\
\hline $\mathrm{CO}_{2}$ & 323 & 250 & 8300 & CDSD-4000 [24] & 0.3 \\
\hline $\mathrm{CO}$ & 194 & 1600 & 6425 & HITEMP 2010 & -1 \\
\hline $\mathrm{HCl}$ & 337 & 50 & 8450 & HITRAN 2012 [25] & -1 \\
\hline
\end{tabular}

For non uniform columns as is the case in the plume, we use further approximations to express the average transmissivity between two abscissa $s_{1}$ and $s_{2}$ along 
a line of sight. The first approximation we may use is the classical Curtis-Godson approximation [26], which consists of averaging the SNB parameters $\bar{k}_{D}, \bar{k}_{L}, \bar{\beta}_{D}$ and $\bar{\beta}_{L}$ over the entire column. The parameters $\bar{k}_{C G}$ and $\bar{\beta}_{C G}$ for a gas species $e$ may be expressed as

$$
\begin{gathered}
\bar{k}_{C G}\left(s_{1}, s_{2}\right)=\frac{1}{u} \int_{s_{1}}^{s_{2}} p_{e}(s) \bar{k}(s) d s, \\
\bar{\beta}_{C G}\left(s_{1}, s_{2}\right)=\frac{1}{u \bar{k}_{C G}\left(s_{1}, s_{2}\right)} \int_{s_{1}}^{s_{2}} p_{e}(s) \bar{k}(s) \bar{\beta}(s) d s,
\end{gathered}
$$

where $u$ is the optical depth of the column.

$$
u=\int_{s_{1}}^{s_{2}} p_{e}(s) d s .
$$

The mean transmissivity between $s_{1}$ and $s_{2}$ is then calculated with the expressions given by Eq. (20), (22) and (23).

The second approximation we may use is the Lindquist-Simmons [27] approximation which provides an approximate value of $\frac{\partial \bar{W}\left(s^{\prime}, s_{2}\right)}{\partial s^{\prime}} d s^{\prime}$ and $\bar{W}\left(s_{1}, s_{2}\right)$ is also calculated as

$$
\frac{\bar{W}\left(s_{1}, s_{2}\right)}{\delta}=-\int_{s_{1}}^{s_{2}} \frac{1}{\delta} \frac{\partial \bar{W}\left(s^{\prime}, s_{2}\right)}{\partial s^{\prime}} d s^{\prime},
$$

where $\frac{\partial \bar{W}\left(s^{\prime}, s_{2}\right)}{\partial s^{\prime}}$ is constructed so that it increases the accuracy of the model for the emission along the optical path at $s^{\prime}$ and transmission to $s_{2}$. Note that $\bar{W}\left(s_{1}, s_{2}\right) / \delta$ is not symmetrical with respect to $s_{1}$ and $s_{2}$ for non uniform path. The spatial derivative expression of $\bar{W}\left(s^{\prime}, s_{2}\right)$ depends on column average parameters $\bar{k}_{C G}\left(s^{\prime}, s_{2}\right)$ and $\bar{\beta}_{C G}\left(s^{\prime}, s_{2}\right)$ and local parameters $k\left(s^{\prime}\right), \beta\left(s^{\prime}\right)$. We use the expressions developed by Young [27] for the Lorentzian broadening and by Rivière and Soufiani [20] for the Doppler broadening. The Lindquist-Simmons approximation is usually more accurate than Curtis-Godson approximation in the case of strong variation of line widths along the optical path, however it consumes more CPU time as there is one more spatial integration over the optical path compared to the Curtis-Godson one.

In order to validate the use of SNB models for the study of high altitude plumes, some comparisons have been made with results obtained with line by line models. Line by line spectra with a resolution of $10^{-3} \mathrm{~cm}^{-1}$ have been calculated using the spectroscopic databases presented in Table 1. These spectra have been tabulated versus the temperature and the pressure following the plume conditions. The tabulation in temperature extends from 100 to $3600 \mathrm{~K}$, with a step of 
$100 \mathrm{~K}$, while the pressure varies from 1.6 Pa to 32 bar, following a geometrical progression with ratio of 2 . For a pressure of $1.6 \mathrm{~Pa}$ the line by line spectra are calculated with pure Doppler broadening line profile whereas for higher pressures the Voigt profile is used.

Comparisons between SNB and line by line models have been made for calculations of radiative transfer along gas columns representative of high altitude plumes. Figure 1 presents pressure and temperature profiles and the intensity incoming from a column starting from the nozzle exit and going along the axis of the plume. Figure 2 (right) shows the intensity incoming, at abscissa $s=0$, from a column crossing the shock wave. The temperature and pressure profiles along this column are shown on Fig. 2 (left).
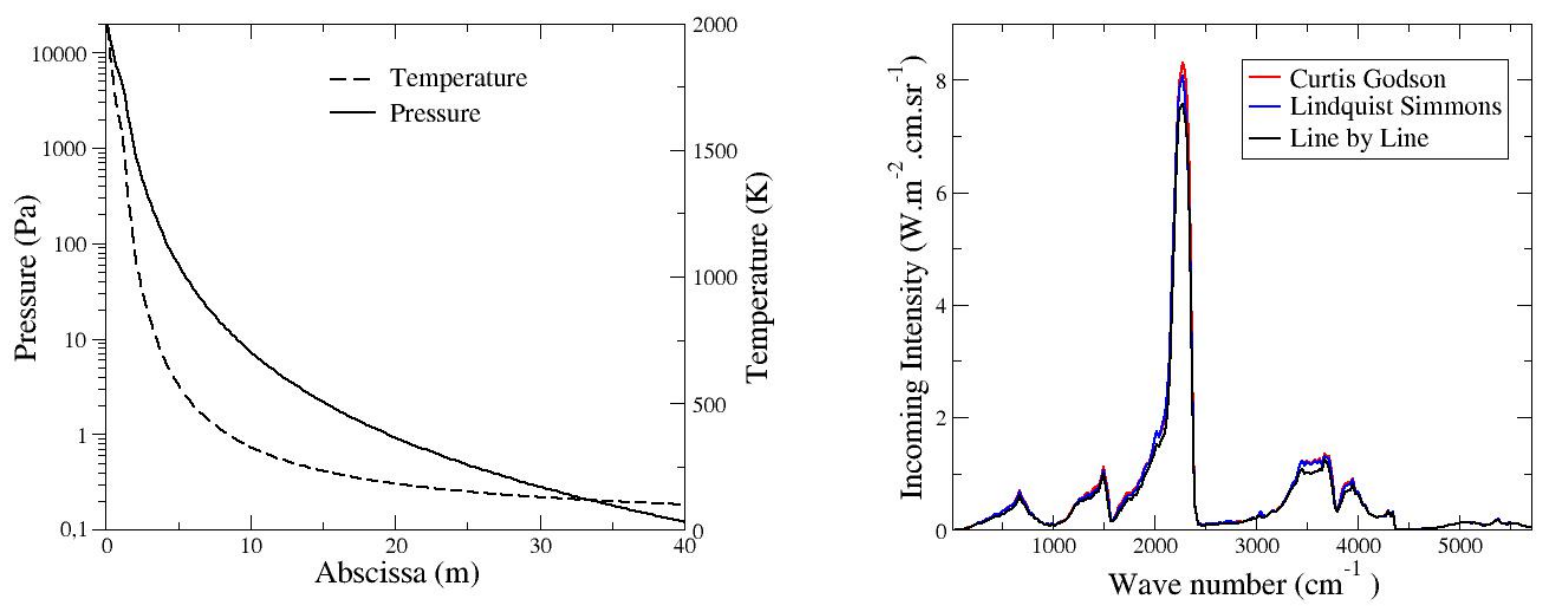

Figure 1: Comparison of gas radiation models for the column along the plume axis; Left: Pressure and temperature profiles along the column; Right: Incoming intensity at abscissa $s=40 \mathrm{~m}$ from the nozzle exit calculated with the different radiation models

In the first case, the averaged relative difference between the intensity calculated with the Curtis-Godson approximation and the reference line by line model is about $9 \%$ whereas it is around $7.5 \%$ using the Lindquist-Simmons approximation. For the second case, differences between SNB results and line by line calculation are higher because of the strong gradients of pressure and temperature along the column and the fact that there are two highly uncorrelated emitting and absorbing zones in the column, a first one is hot with relatively low pressure and a second 

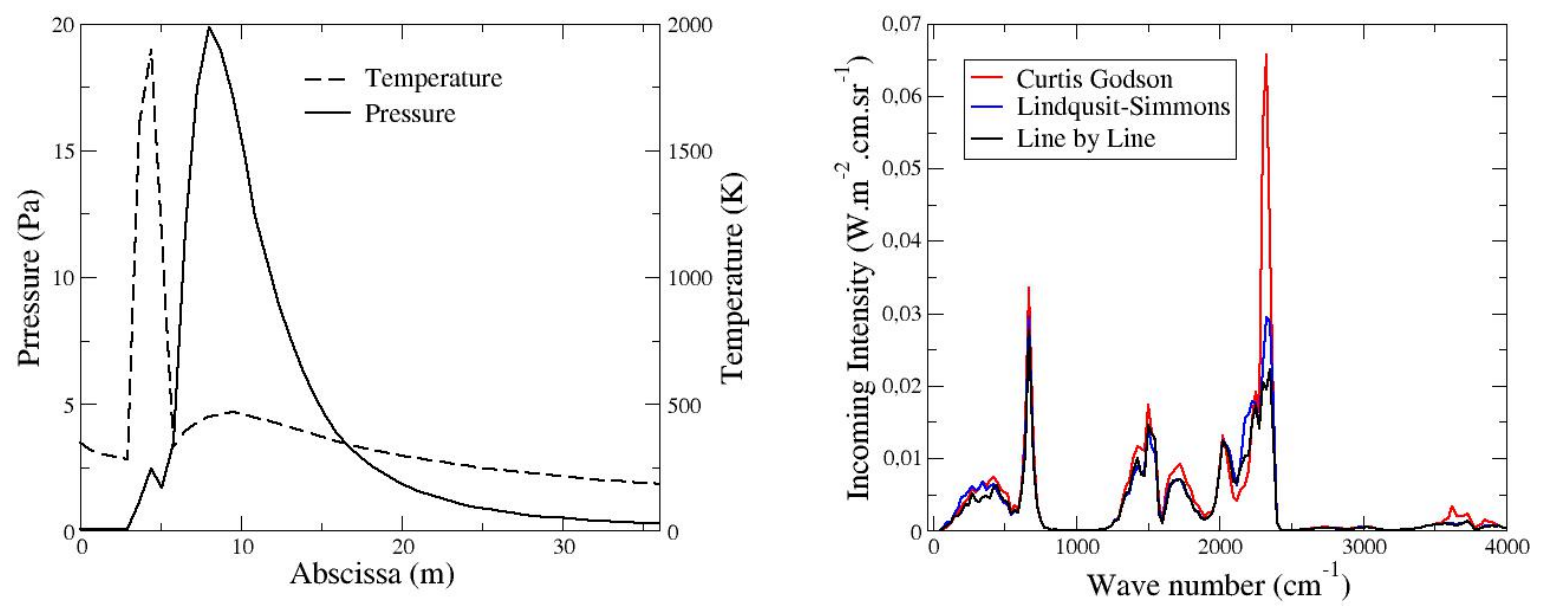

Figure 2: Comparison of gas radiation models for a column crossing the shock wave of the plume; Left: Pressure and temperature profiles along the studied column; Right: Incoming intensity, at abscissa $s=0 \mathrm{~m}$, calculated with the different radiation models

one colder but with higher pressure. Relative differences are about $13 \%$ for the Linquist-Simmons model and 21.5\% for the Curtis-Godson model. Among different tested lines of sight in the plume, this case leads to the less accurate results.

These comparisons show that the SNB models are appropriate for the study of gas radiation in high altitude plumes, as they have a relatively good accuracy compared to line by line calculations, especially given the high non-uniformity and gradients of temperature and pressure. As expected, the Lindquis-Simmons approximation has been shown to give more accurate results than the Curtis-Godson one.

\subsection{Radiative transfer modeling}

We consider first the case of a non-scattering medium containing $N_{s}$ different emitting species. The monochromatic radiative transfer equation along an optical path can be written, under its integral form

$$
I_{\sigma}(s)=\sum_{j=1}^{N_{s}} \int_{s_{0}}^{s} \eta_{\sigma}^{j}\left(s^{\prime}\right) \prod_{j^{\prime}=1}^{N_{s}} \tau_{\sigma}^{j^{\prime}}\left(s^{\prime}, s\right) d s^{\prime},
$$

where $s$ is the abscissa along the optical path, $I_{\sigma}(s)$ is the radiative intensity incoming at $s, \eta_{\sigma}^{i}(s)$ the spectral emission coefficient of the species $j$ and $\tau_{\sigma}^{j}\left(s^{\prime}, s\right)$ the monochromatic transmissivity of the optical path $\left(s^{\prime}, s\right)$ due to species $j$. In 
our study the $N_{s}$ species are the $N_{m}$ molecular species and the $N_{c}$ particle classes. When averaging the previous equation over a spectral band $\Delta \sigma$, a distinction has to be made between the contributions of the gases, for which the absorption may be strongly correlated to the emission, and the contributions of particles. Then the averaged intensity may be written as the sum of two contributions,

$$
\overline{I_{\sigma}(s)}=\overline{I_{\sigma}^{g a s}(s)}+\overline{I_{\sigma}^{p a r t}(s)} .
$$

For non-optically thin media, assuming that there is no spectral correlation between the spectra of the different molecular species, the radiative intensity $\overline{I_{\sigma}^{\text {gas }}(s)}$ due to gas emission can be written from Eq. (26) as [28]

$$
\overline{I_{\sigma}^{g a s}(s)}=\sum_{j_{g a s}=1}^{N_{m}} \int_{s_{0}}^{s}\left[\overline{\frac{\eta_{\sigma}^{j_{g a s}}\left(s^{\prime}\right)}{\kappa_{\sigma}^{j_{g a s}}\left(s^{\prime}\right)}} \frac{\overline{\partial \tau_{\sigma}^{j_{g a s}}\left(s^{\prime}, s\right)}}{\partial s^{\prime}} \prod_{j^{\prime}=1, j^{\prime} \neq j_{g a s}}^{N_{s}} \overline{\tau_{\sigma}^{j^{\prime}}\left(s^{\prime}, s\right)}\right] d s^{\prime} .
$$

At local thermal equilibrium, the ratio between the emission and the absorption coefficient of the $j^{\text {th }}$ species $\eta_{\sigma}^{j}(s) / \kappa_{\sigma}^{j}(s)$ can be replaced by the Planck's function $I_{\sigma}^{0}$ at the temperature of species $j$ thanks to the Kirchhoff's Law. As all the gas species are assumed at the same temperature and because their spectra are considered non correlated, the sum over the $N_{m}$ species can be simplified given the expression of the gas transmissivity $\tau_{\sigma}^{g}$

$$
\overline{\tau_{\sigma}^{g}\left(s, s^{\prime}\right)}=\prod_{j=1}^{N_{m}} \overline{\tau_{\sigma}^{j}\left(s, s^{\prime}\right)} .
$$

Equation (28) then becomes

$$
\overline{I_{\sigma}^{g a s}(s)}=\int_{s_{0}}^{s}\left[\overline{I_{\sigma}^{0}\left(T_{g}\left(s^{\prime}\right)\right)} \frac{\partial \overline{\tau_{\sigma}^{g}\left(s^{\prime}, s\right)}}{\partial s^{\prime}} \prod_{j_{\text {part }}=1}^{N_{c}} \overline{\tau_{\sigma}^{j_{\text {part }}}\left(s^{\prime}, s\right)}\right] d s^{\prime} .
$$

For the particles, the emission coefficient $\eta_{\sigma}^{j}(s)$ and the absorption coefficient $\kappa_{\sigma}^{j}(s)$ slowly vary over the band $\Delta \sigma$ and can be considered as constant, the expression of the incoming intensity $\overline{I_{\sigma}^{\text {part }}(s)}$ produced by the particles along the optical path can then be expressed from Eq. (26) as

$$
\overline{I_{\sigma}^{p a r t}(s)}=\sum_{k=1}^{N_{c}} \int_{s_{0}}^{s}\left[\overline{\eta_{\sigma}^{k}\left(s^{\prime}\right)} \prod_{j^{\prime}=1}^{N_{s}} \overline{\tau_{\sigma}^{j^{\prime}}\left(s^{\prime}, s\right)}\right] d s^{\prime} .
$$


In practice, we use a Monte Carlo method [29] to calculate radiative fluxes and volumetric powers integrated over all the directions $\Omega$ and over the spectrum. In a first step, the emitted power is calculated in each volume element $i$ as the sum of the gas and the particle emissions over the whole spectrum :

$P_{\text {emi }}^{i}=4 \pi V_{i} \sum_{\text {band } \Delta \sigma}\left[\sum_{j_{g a s}=1}^{N_{m}} \overline{\kappa_{i}^{j_{g a s}}} \overline{I_{\sigma}^{0}\left(T_{g}(i)\right)}+\sum_{k=1}^{N_{c}} \overline{\kappa_{i}^{(k)}} \overline{I_{\sigma}^{0}\left(T_{p}^{(k)}(i)\right)}\right] \Delta \sigma=P_{\text {emi,gas }}^{i}+P_{\text {emi,part }}^{i}$,

where the factor $4 \pi$ stands for the direction integration, $V_{i}$ is the volume of the element $i$ and $\Delta \sigma$ is the band width equal to $25 \mathrm{~cm}^{-1}$ over the SNB model spectrum range (50 to $11250 \mathrm{~cm}^{-1}$ ), and equal to $200 \mathrm{~cm}^{-1}$ for the calculation of particle radiation beyond the IR range (from 11250 to $51250 \mathrm{~cm}^{-1}$ ).

In a second step, the Monte Carlo algorithm calculates the absorption power for each volume element. In order to do this, the emission power of each volume element $i$ is divided into $N_{i}$ energy bundles. Each bundle emitted in the cell $i$ is first associated with a source point in the cell and a direction, both randomly generated. Then the spectral band is randomly chosen according to the probability distribution function of the spectral emission in cell $i$. Once the bundle is characterized, it follows an optical path through the mesh, leaving energy by absorption in every cell $i^{\prime}$ it passes through, until the carried power is less than a cutoff criterion.

The absorption power $P_{a b s, i^{\prime}}^{i}$ of a bundle emitted in a cell $i$ and absorbed in a cell $i^{\prime}$ is calculated depending on the emitting species.

The absorbed power $P_{a b s, i^{\prime}}^{i, g a s}$ due to gas emission in the cell $i$ is calculated, according to the discretized form of Eq. (30), using the following expression

$$
\begin{aligned}
P_{a b s, i^{\prime}}^{i, g a s}= & \frac{P_{\text {emi,gas }}^{i}}{N_{i} \overline{\kappa_{i}^{g}} \Delta s}\left[\left(\overline{\tau^{g}}\left(s_{i}+\Delta s, s_{i^{\prime}}^{-}\right)-\overline{\tau^{g}}\left(s_{i}, s_{i^{\prime}}^{-}\right)\right) \prod_{k=1}^{N_{c}} \overline{\tau^{(k)}\left(s_{i}, s_{i^{\prime}}^{-}\right)}\right. \\
& \left.-\left(\overline{\tau^{g}}\left(s_{i}+\Delta s, s_{i^{\prime}}^{+}\right)-\overline{\tau^{g}}\left(s_{i}, s_{i^{\prime}}^{+}\right)-\right) \prod_{k=1}^{N_{c}} \overline{\tau^{(k)}\left(s_{i}, s_{i^{\prime}}^{+}\right)}\right],
\end{aligned}
$$

where $s_{i}, s_{i^{\prime}}^{-}, s_{i^{\prime}}^{+}$are respectively the abscissa of the source point, and the inlet and outlet points of the absorbing cell $i^{\prime}$, and $\Delta s$ a length of $10^{-6} \mathrm{~m}$ small enough to consider the optical path along $\Delta s$ optically thin. $\overline{\kappa_{i}^{g}}$ is the total gas absorption coefficient averaged over $\Delta \sigma$, calculated from band model parameters.

The absorbed power $P_{a b s, i^{\prime}}^{i, p a r t}$ due to particle emission is calculated following Eq. 
(31) as

$$
P_{a b s, i^{\prime}}^{i, p a r t}=\frac{P_{\text {emi,part }}^{i}}{N_{i}}\left[\prod_{j=1}^{N_{s}} \overline{\tau^{j}\left(s_{i}, s_{i^{\prime}}^{-}\right.}{ }^{\Delta \sigma}-\prod_{j=1}^{N_{s}}{\overline{\tau^{j}\left(s_{i}, s_{i^{\prime}}^{+}\right)}}^{\Delta \sigma}\right] .
$$

The global absorption of the radiation emitted in the cell $i$ and absorbed by $i^{\prime}$ for this bundle is then the sum of $P_{a b s, i^{\prime}}^{i, g a s}$ and $P_{a b s, i^{\prime}}^{i, p a r t}$. In order to evaluate the radiative source terms for the gas phase and for each class of the particulate phase, needed in Eqs. (2) and (7) for the coupling of the radiation with the flow field, a splitting of the absorbed power for each contribution is made. So, the part of the radiation power absorbed by a species $j$ (gas or a class of particle) in the cell $i^{\prime}$ $P_{a b s, i^{\prime}}^{i}(j)$ is calculated as follows

$$
P_{a b s, i^{\prime}}^{i}(j)=\frac{\widetilde{\kappa_{i^{\prime}}(j)}}{\sum_{j^{\prime}=1}^{N_{s}} \widetilde{\kappa_{i^{\prime}}\left(j^{\prime}\right)}} P_{a b s, i^{\prime}}^{i, g a s}+\frac{\widehat{\kappa_{i^{\prime}}(j)}}{\sum_{j^{\prime}=1}^{N_{s}} \widehat{\kappa_{i^{\prime}}\left(j^{\prime}\right)}} P_{a b s, i^{\prime}}^{i, p a r t},
$$

where $\widetilde{\kappa_{i^{\prime}}(j)}$ and $\widehat{\kappa_{i^{\prime}}(j)}$ are the equivalent absorption coefficients of the $j^{\text {th }}$ species in the cell $i^{\prime}$ for both contributions, equal to the effective absorption coefficient ${\overline{K^{\prime},(k)}}^{\Delta \sigma}$ if $j$ is the $k^{\text {th }}$ class of particles and calculated as follows for gases:

$$
\begin{gathered}
\kappa_{i^{\prime}}(\text { gas })=\frac{1}{\left|s_{i^{\prime}}^{-}, s_{i^{\prime}}^{+}\right|} \ln \left(\frac{\overline{\tau^{g}}\left(s_{i}+\Delta s, s_{i^{\prime}}^{-}\right)-\overline{\tau^{g}}\left(s_{i}, s_{i^{\prime}}^{-}\right)}{\overline{\tau^{g}}\left(s_{i}+\Delta s, s_{i^{\prime}}^{+}\right)-\overline{\tau^{g}}\left(s_{i}, s_{i^{\prime}}^{+}\right)}\right), \\
\left.\kappa_{i^{\prime}} \widehat{(g a s}\right)=\frac{1}{\left|s_{i^{\prime}}^{-}, s_{i^{\prime}}^{+}\right|} \ln \left(\frac{\overline{\tau^{g}\left(s_{i}, s_{i^{\prime}}^{+}\right)}}{\overline{\tau^{g}\left(s_{i}, s_{i^{\prime}}^{-}\right)}}\right),
\end{gathered}
$$

where $\left|s_{i^{\prime}}^{-}, s_{i^{\prime}}^{+}\right|$is the length between the inlet and the outlet points of cell $i^{\prime}$. The total radiative power $P_{r a d, j}^{i}$ of the species $j$ in the finite volume $i$, integrated over the whole spectrum, is then expressed as

$$
P_{r a d, j}^{i}=-P_{e m i, j}^{i}+\sum_{i^{\prime}} P_{a b s, i}^{i^{\prime}}(j)
$$

The above sum extends over all the bundles issued from $i^{\prime}$ and crossing the volume $i$.

With the Curtis-Godson approximation, the transmissivities $\overline{\tau^{g}\left(s_{i}, s_{i^{\prime}}\right)}$ can easily be calculated from Eq. (22) and Eq. (23). Using the Lindquist-Simmons (LS) approximation with the forward method (FM) previously described (the bundles go from the emitting cell to an absorbing cell) would be really costly in CPU time and computational memory because to calculate $\overline{\tau_{L S}^{g}\left(s_{i}, s_{i^{\prime}}\right)}$, integration in Eq. (25) 
is required from the absorbing point $i^{\prime}$ until the emission point $i$, that is to say we need to know all the physical properties of the gas phase between $i$ and $i^{\prime}$, and perform the integration of Eq. (25) for each new absorbing cell of the optical path.

In order to use the Lindquist-Simmons approximation, a Monte-Carlo reciprocal method has been developed. The method is the same as the forward approach but, this time, the bundles start from the absorbing cell $i$ and go through emitting cells $i^{\prime}$ (Reciprocal Method RM). The emitted power of the volume element $i$ for each emitting species is calculated by a deterministic manner as in Eq. (32). To calculate the absorbed power, we use the fact that the emission terms $\overline{\eta_{\sigma}^{j}\left(s^{\prime}\right) / \kappa_{\sigma}^{j}\left(s^{\prime}\right)}$ of Eq. (28) are not correlated with the transmission terms. As a consequence, the power absorbed by a species $j$ in the cell $i$ of the radiation emitted by a species $j^{\prime}$ in the cell $i^{\prime} \overline{P_{a b s, j, i}^{e m i, j^{\prime}, i^{\prime}}}$ can be expressed as a function of the forward calculated power absorbed by the species $j^{\prime}$ in the cell $i^{\prime}$ and emitted by the species $j$ of the cell $i \overline{P_{a b s, j^{\prime}, i^{\prime}}^{e m i, j,}}$ according to the following expression

$$
\overline{P_{a b s, j, i}^{e m i, j^{\prime}, i^{\prime}}}(R M)=\overline{P_{a b s, j^{\prime}, i^{\prime}}^{e m i, j, i}}(F M) \frac{\overline{I_{\sigma}^{0}\left[T_{j^{\prime}}\left(i^{\prime}\right)\right]}}{\overline{I_{\sigma}^{0}\left[T_{j}(i)\right]}} .
$$

The developed reciprocal method not only enables us to use the LindquistSimmons approximation to solve the radiative transfer equation with a quite reasonable amount of CPU time, it also allows us to evaluate radiative power and radiative fluxes at given points of the mesh. Indeed, contrary to the forward method for which we need to launch bundles from every part of the volume to know the radiative quantities at a given point, with the reciprocal methods, we only need to launch rays from this point to have the information.

The radiative solver also takes into account the scattering by particles. In order to do that, a length of scattering is randomly chosen depending on the scattering coefficient of the particles, then the new direction followed by the bundle is randomly generated according to the phase function. The influence of the scattering on the radiative transfer will be discussed in Sec. 4.2.

\section{Results}

The methods described in the previous sections were employed to carry out numerical simulations of high altitude solid propellant rocket plumes. In particular, we focus in the following on the simulation of the plume flow field corresponding to the second stage, Antares II motor burn, of the Strypi IX rocket 
launched in 1991. For this launch the "Bow Shock Ultraviolet 2" experiment was conducted by Erdman et al. [5], measuring the spectral intensity emitted by the exhaust plumes in the UV range, for wavelengths between 0.2 and $0.4 \mu \mathrm{m}$, using photometers and spectrometers mounted on the front of the vehicle. For both the second stage Antares II motor and the third stage Star 27 motor, two measurements of the spectral radiance have been done for different times-after-launch.

The simulation presented here corresponds to a time-after-launch of $184 \mathrm{sec}$ of the experiment BSUV2, for which the solid propellant motor Antares II was flying at an altitude of $109.6 \mathrm{~km}$ with a flight speed of $1.97 \mathrm{~km} / \mathrm{sec}$. We chose to first work on the Antares II motor rather than the Star 27 one, because its plume is more emissive and also because the outside pressure is higher than that of the Star 27 flight, which makes more relevant the use of a Navier-Stokes code to solve the gas flow field.

After a presentation of the simulation setup, the results of the simulation of the Antares II plume flow field will be shown and comparisons will be made with the experimental data and other work done on the simulation of this plume. For the simulation of gas radiation, we used here the Curtis-Godson approximation, less costly in CPU time.

\subsection{Simulation setup}

To simplify the simulation, and to limit the computational requirements, the plume is assumed to be axisymmetric and at steady state. The flow is also assumed non reactive and non turbulent, which is reasonable because of the low density conditions. Using the information given by Smather et al [30], the Antares II vehicle is represented by a cone-cylinder shape of $5.87 \mathrm{~m}$ length. The nozzle has an exit diameter of $0.78 \mathrm{~m}$, and a cone half angle of $15^{\circ}$. We use slip wall boundary conditions for the wall of the vehicle whereas the inflow boundaries of the nozzle start at the exit plane of the nozzle. Since we use a 3D radiative solver, to decrease the CPU time the global grid is represented by an axisymmmetric sector of $9^{\circ}$, extending $10 \mathrm{~m}$ upstream to $60 \mathrm{~m}$ downstream of the nozzle exit and with a radius of $30 \mathrm{~m}$ from the axis of symmetry. The mesh is composed of 850000 unstructured cells, refined close to the nozzle. A plane section of the computational domain is shown in Fig. 3.

For the inflow boundaries of the nozzle exit, since little information is given in the literature, a typical composition of the exhaust gas for the Antares motor, as well as the mass fraction of alumina particles which accounts for $37.5 \%$ of the total mass flow at the nozzle exit, from Smather et al [30] are used. The global composition of the exhaust gases leaving the nozzle is given in Table 2. 


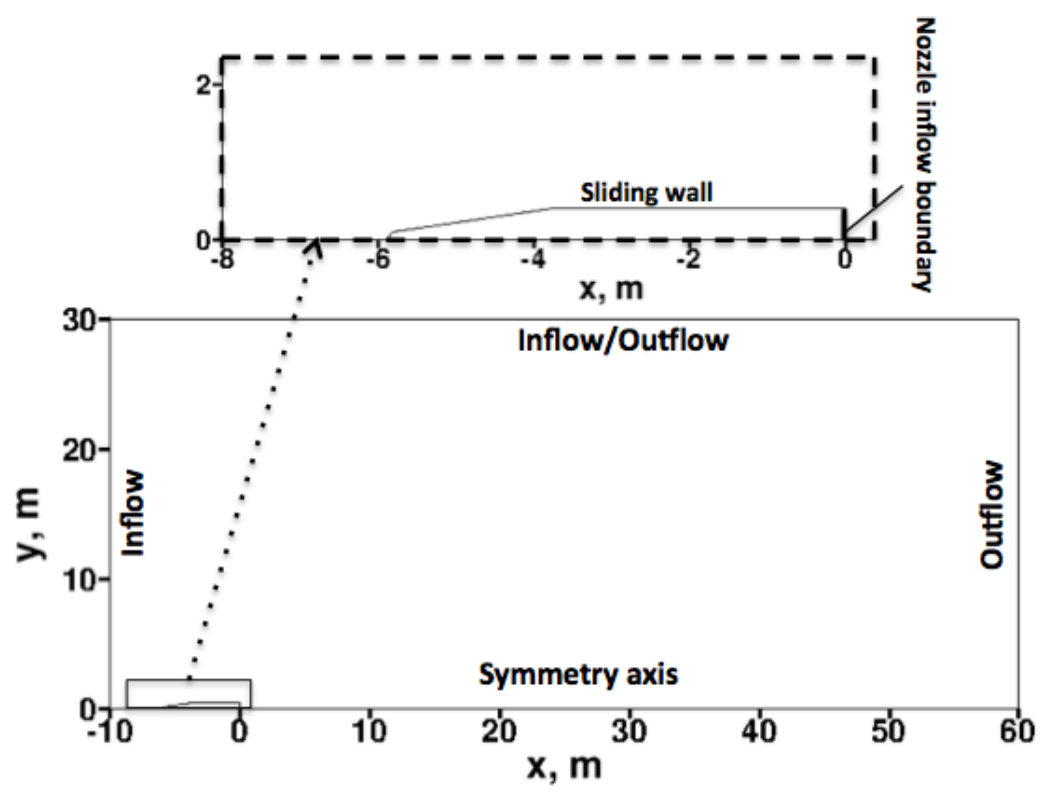

Figure 3: Schematic representation of the domain used for simulations of the Antares II plume

For the flow conditions of both gas phase and condensed phase, we use the data

Table 2: Composition of the exhaust gases

\begin{tabular}{l|ccccccc} 
Species & $\mathrm{CO}_{2}$ & $\mathrm{CO}$ & $\mathrm{H}_{2} \mathrm{O}$ & $\mathrm{HCl}$ & $\mathrm{N}_{2}$ & $\mathrm{H}_{2}$ & $\mathrm{H}$ \\
\hline Mass fraction & $3.0410^{-2}$ & 0.6235 & $4.9210^{-2}$ & $3.5210^{-2}$ & 0.23 & $3.1510^{-2}$ & $310^{-4}$
\end{tabular}

of Candler and al. [2] given in Table 3. Note that the global mass flow rate at the nozzle exit is in agreement with the average thrust given by Smather et al [30]. The velocity direction of gas and particles varies linearly with the distance to the axis. The velocity is along the axis of symmetry in the center of the nozzle whereas it has an angle of $15^{\circ}$ near the lip. The other properties are assumed to be constant over the whole nozzle exit plane. For the size distribution of alumina particles, three different classes have been used whose diameters vary from 1 to 8 $\mu \mathrm{m}$. Table 4 shows the particle size distribution among the three different classes and their respective mass fraction. In their study, Candler and al. [2] distributed the particles over five classes. We chose to represent only three classes, a small one, a medium one and a large one in order to decrease the CPU time. This different modeling may cause small differences between our results. However, many other modelling aspects are expected to produce more important differences between the results of Ref. [2] and our results. Indeed, supercooling phenomenon, 
as well as absorption by particles were not considered in [2], while emission by particles was based on simplified models rather than on Mie theory in their study.

In order to take into account the radiation from the hot nozzle walls and from gas/particle mixture inside the nozzle, the boundary inlet section was modeled as a gray opaque surface of emissivity 0.5 and at a temperature equal to $2100 \mathrm{~K}$, close to gas inlet temperature.

The upstream atmospheric boundary conditions shown in Fig. 3 are composed of a gas mixture with a mass fraction of 0.75 for $\mathrm{N}_{2}$ and 0.25 for $\mathrm{O}_{2}$, a temperature of $260 \mathrm{~K}$, a density of $6.6910^{-7} \mathrm{~kg} \cdot \mathrm{m}^{-3}$ and a velocity equal to the vehicle speed of $1.97 \mathrm{~km} . \mathrm{s}^{-1}$, parallel to the symmetry axis.

Table 3: Flow conditions at the nozzle exit

\begin{tabular}{|c|c|c|}
\hline & gas & particles \\
\hline${\text { Velocity }\left(\mathrm{km} . \mathrm{s}^{-1}\right)}^{2}$ & 2651 & 2651 \\
$\rho(\mathrm{kg} \cdot \mathrm{m}-3)$ & 0.020915 & 0.012428 \\
$\mathrm{~T}(\mathrm{~K})$ & 2139 & 2320 \\
\hline
\end{tabular}

Table 4: Particle sizes and mass fraction distribution used in the simulation

\begin{tabular}{|c|c|c|}
\hline class & $\mathrm{D}_{p} \mu \mathrm{m}$ & mass fraction \\
\hline 1 & 1 & 0.1 \\
2 & 4 & 0.7 \\
3 & 8 & 0.2 \\
\hline
\end{tabular}

\subsection{Simulation Results and Comparisons}

Figure 4 presents the temperature and pressure profiles of the gas phase for a part of the simulated flow field. We can first notice the important expansion of the exhaust gases as the pressure and temperature rapidly decrease downstream from the nozzle. Moreover, we may observe the large diffuse bow-shock induced by the shock of the atmosphere with both the vehicle and the plume. A small back-flow region appears on above the nozzle lip. There, the continuum approach used for the simulation of the gas phase may be deficient and could impact the profile of the bow-shock but would not affect the main core of the plume.

The temperature profiles of the different classes of particles are presented in Fig. 5. The upper part of the axis $(Y>0)$ shows the simulation results without radiation (only the convective transfer with the gas affects the cooling of particles), whereas the lower part shows the results using the radiative coupling with the flow 

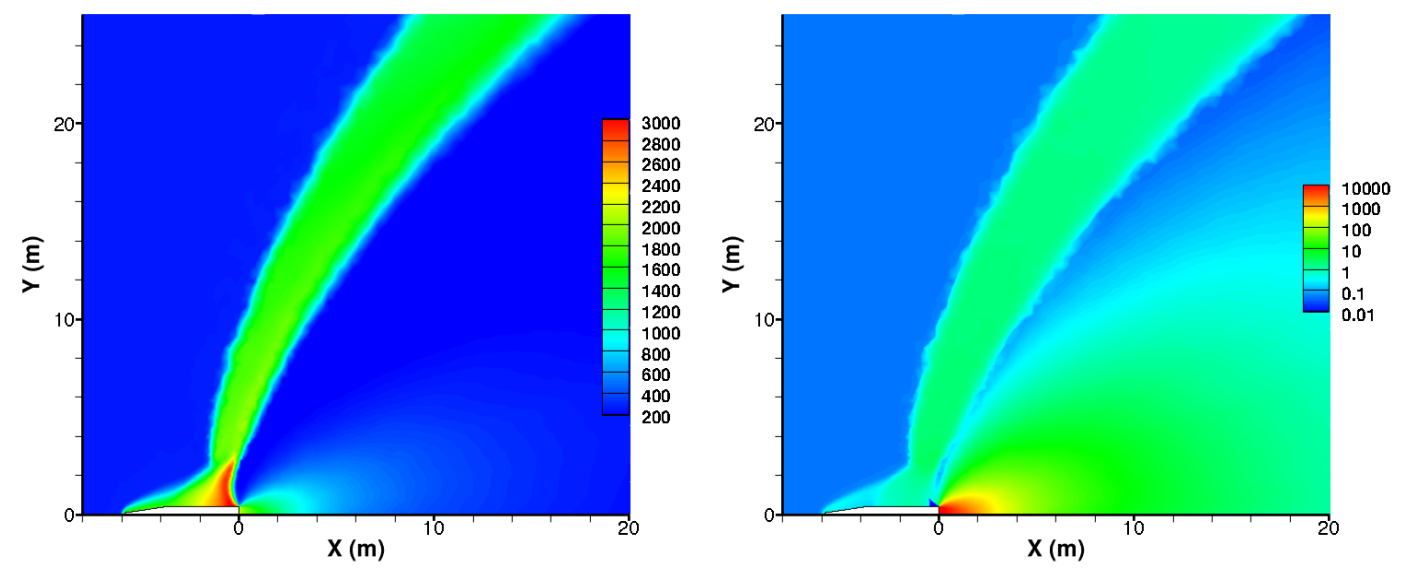

Figure 4: Gas-phase flowfield for the Antares II burn, Left: Temperature profile in K; Right: Pressure profile in $\mathrm{Pa}$

field. We may first notice the difference in the expansion of particles between the different size classes. Indeed the smallest particles better follow the expansion of the gas due to the fact that momentum exchange between gas and particles (same for the convective exchange) is approximately proportional to $\mathrm{D}_{p}^{2} / \mathrm{D}_{p}^{3}$. The smallest particles, of $1 \mu \mathrm{m}$ diameter, experience a rapid cooling along the expansion of the plume because of the important convective heat transfer, and quickly solidify (about $3 \mathrm{~m}$ after the nozzle exit). The radiative transfer does not greatly influence the cooling of small particles as the internal energy losses are mainly driven by the convective transfer (see Fig. 6). For larger particles, the radiative transfer plays a major role for the establishment of the temperature. Indeed, without radiation, both 4 and $8 \mu \mathrm{m}$ size particles have not started to solidify in the domain shown. With the radiative coupling, the phase change, associated with the jump in temperature due to the supercooling phenomenon, starts about $5 \mathrm{~m}$ downstream from the nozzle exit for medium size particles and $58 \mathrm{~m}$ downstream for the largest ones. Moreover, for all particle classes, the temperature increases with the distance from the axis according to the fact that the convective transfer, which strongly impacts the cooling at the beginning of the expansion, decreases as we move away from the axis, since the gas density becomes very small.

Figure 6 presents the evolution of the volumetric power loss due to convective and radiative transfers for each class of particles along the axis of the plume. It shows that the cooling of the smallest particles is strongly driven by the convective exchange with the gas phase, whereas, for the larger ones, the radiative loss 

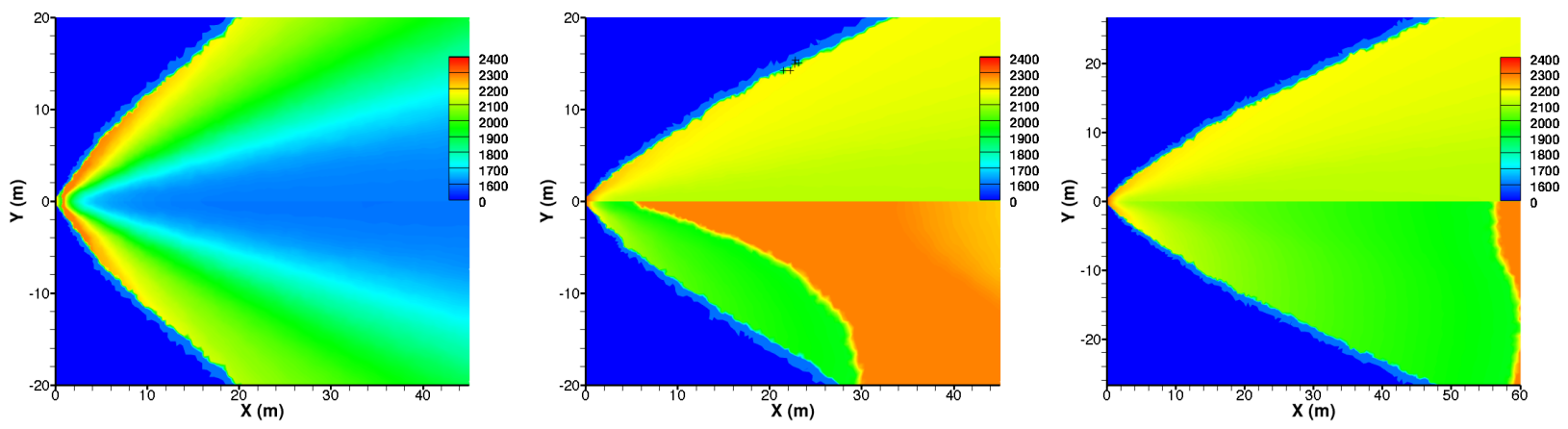

Figure 5: Influence of the radiative coupling on the temperature of the different particle classes. Above the axis: Temperature calculated without radiative coupling. Below: With coupling. Left: $1 \mu \mathrm{m}$ diameter, Center: $4 \mu \mathrm{m}$ diameter, Right: $8 \mu \mathrm{m}$ diameter

exceeds the convective loss after about $10 \mathrm{~m}$ from the nozzle exit. Note finally that the radiative coupling does not affect significantly the gas conditions, as it only reduces its temperature by a few degrees (about $3 \mathrm{~K}$ ), the quick expansion ruling its evolution along the plume.

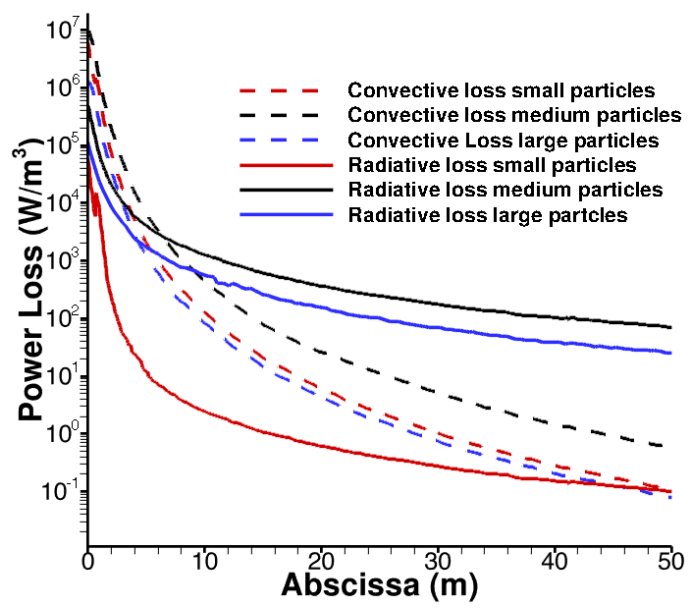

Figure 6: Comparison between convective and radiative power losses for particles along the plume axis. Coupled calculations.

In order to validate the modeling of radiation for high altitude plumes and to compare the influence of the different simulation parameters, comparisons have been made with the onboard measurements made during the Strypi IX experiments [5], corresponding to a time after launch of $184 \mathrm{~s}$ during the Antares II motor burn. The line of sight in the experiment pointed downstream with a $4^{\circ}$ angle 
with respect to the axis of the plume and had a $4^{\circ}$ field of view. The spectrometer measured the spectral intensity incoming from the plume in the UV spectral range, for wavelengths between 0.2 to $0.4 \mu \mathrm{m}$ where only the particles contribute to radiation with our modeling.

The comparison of the results with experimental data is shown on Fig. 7. The baseline simulation corresponds to results obtained with a three-way coupling simulation, involving the scattering by the particles. The uncoupled simulations correspond to a radiation calculation performed without taking into account the coupling of the radiative transfer with the two-phase flow field. Published numerical results of Candler et al [2] are also presented oin Fig. 7.

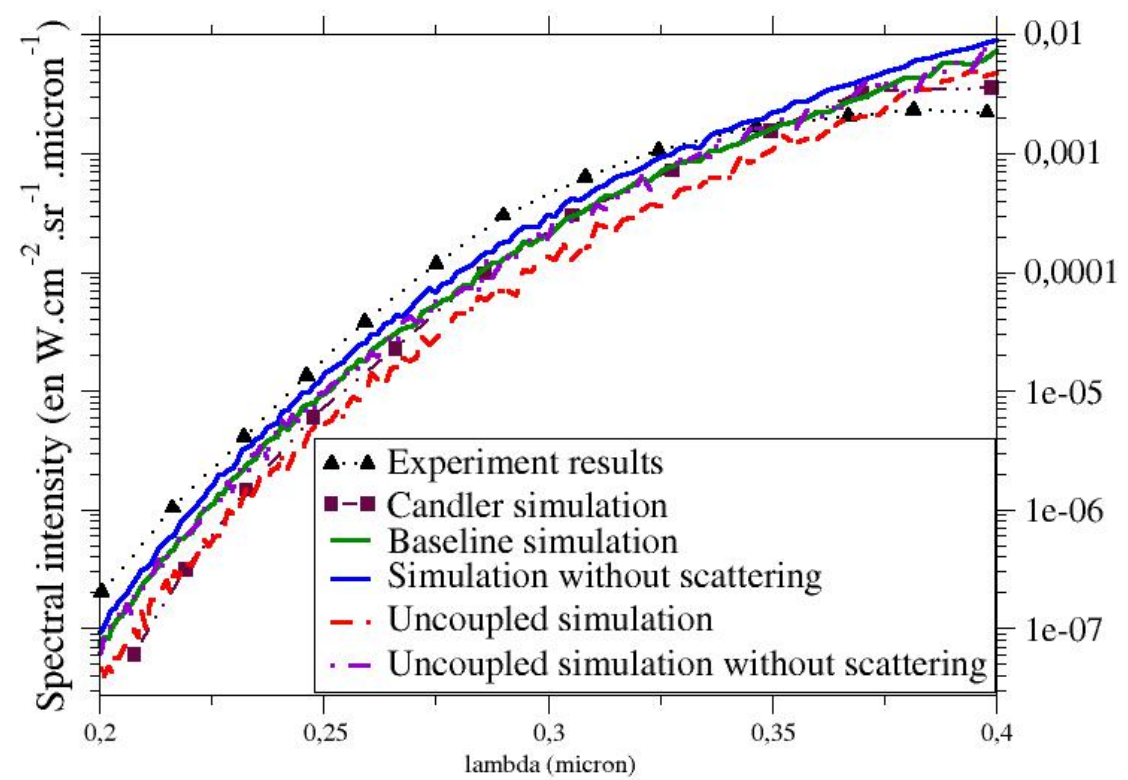

Figure 7: Experimental measurements and simulation results of the UV spectral intensity detected by the on-board spectrometer

The baseline simulation results relatively well reproduce the shape of the experimental measurements. However, below $0.35 \mu \mathrm{m}$, the calculated spectral intensities underestimate the experimental data by a factor around 2. Note that in this region of the spectrum the obtained results are better than those calculated by Candler et al. [2] from who the flow field conditions were taken. Many factors may explain these discrepancies with the experiment. The first of all is the uncertainty on the spectral and temperature dependence of alumina absorption index which 
may strongly influence the radiation calculation. Indeed, the absorption index has been proven to strongly depend on the impurities present in the combustion produced alumina [14]. Secondly, the uncertainty and approximations made on the nozzle exit conditions would also play an important role on the results. Simulating the two-phase flow field starting from inside the nozzle could improve the accuracy of the simulations. Besides, we may add the fact that our simulation domain ends $60 \mathrm{~m}$ downstream from the nozzle, which does not influence the flow field simulation but prevents from taking into account the radiation emitted by farther regions of the flow field.

Given the differences with the experimental measurements, the comparison between the various conditions of simulation reveals the impact of the different models used for the simulation of radiation in high altitude plumes. First, Fig. 7 shows that the scattering by particles tends to decrease the spectral intensity detected by the spectrometer in the UV range (this is not necessarily the case for all spectral ranges and for other lines of sight), screening the radiation by increasing the optical path between the source and the detector. Besides, the comparison between the coupled and uncoupled simulations shows that the coupling of the flow field with radiation increases the spectral intensity. At first glance, we would expect that the coupling with radiation tends to decrease the spectral intensity as it speeds up the cooling along the expansion of the plume but, actually, the opposite behavior occurs due to the phase change phenomenon. First, increasing the cooling of liquid particles by radiation speeds up their phase transition, and, due to the supercooling phenomenon, induces a jump to the melting temperature. This leads to an increase of particles temperature which is strongly correlated with their emission. This is especially the case for the medium sized particles (see Fig. 5 ), which correspond to $70 \%$ of the particle mass flow rate. The other reason is because speeding up the solidification raises the mass fraction of the solid phase, which has a greater absorption index than the liquid phase in the UV range.

There are unfortunatly no experimental data available in the infrared region which corresponds to most of the emission from the plume. Figure 8 presents the simulated spectral intensity incident on the spectrometer for the whole spectrum. According to the simulations, the intensity in the UV range counts for less than 0,2 $\% o$ of the global incoming intensity. Figure 8 shows that for this optical path, the scattering tends to decrease the incoming intensity by about $20 \%$ in most of the spectrum except in the less far IR range where scattering is very small. According to the temperature distribution shown in Fig. 5, emission is mostly due to the hot medium sized particles far from the spectrometer, in the region corresponding to the crystallization, and it is screened by colder liquid particles closer to the 
spectrometer. Besides, we see on this figure some spectral regions with important contribution of gas emission (for example $\mathrm{CO}_{2}$ near $2300 \mathrm{~cm}^{-1}$ ) which shows that gas radiation must be taken into account in the coupled simulations.

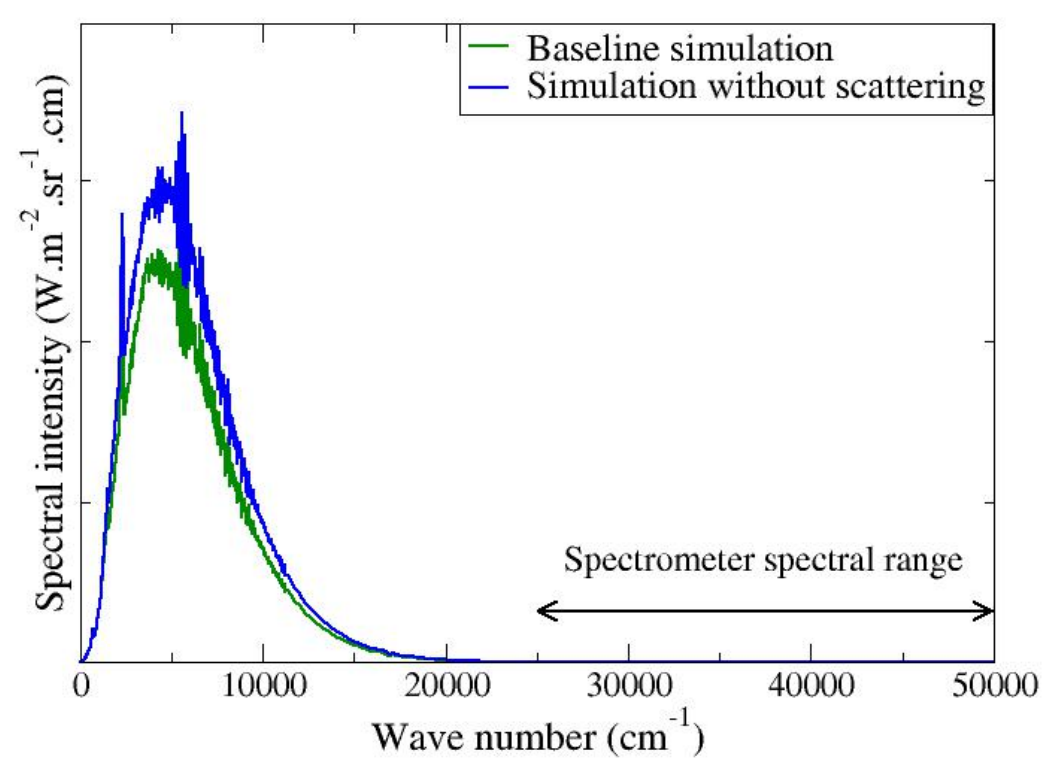

Figure 8: Incident spectral intensity on the spectrometer in the whole spectrum

\section{Conclusions}

The simulation of radiation in solid propellant exhaust plumes at high altitude requires the modeling of several physical processes which include the gas/particles interactions in rarefied flows, the supercooling of alumina and radiative properties of both phases. Even if many approximations are assumed to simplify the study of high altitude plumes, the work presented here introduces different models in order to better take into account these physical processes for the simulation of plume flow field and radiation. The numerical scheme developed enables to carry out fully coupled simulations of high altitude solid rocket plume flows and to evaluate radiation within the plume. Comparisons between experimental measurements of plume radiation and numerical calculations have been made. They show relatively good agreement between simulation and experimental results given the known sources of uncertainties. Furthermore, the study of 
the influence of radiation on the flow field has shown that radiative transfer plays an important role for an accurate description of plume conditions, especially the temperature and the phase state of alumina particles along the plume. The presented computational method may provide a tool for the calculation of heat flux on the walls of rockets and to obtain accurate flow field conditions, necessary for the analysis of high altitude plume signatures.

\section{Acknowledgments}

The present research was done thanks to a $\mathrm{PhD}$ Grant from Délégation Générale de l'Armement, French Ministry of Defense. The computation of gas radiation spectra was performed using HPC resources from GENCI-IDRIS (Grant 2017-A0022B00209).

\section{Bibliography}

[1] F. S. Simmons, Rocket Exhaust Plume Phenomenology, El Segundo, AerospacePress, CA, 2000.

[2] G. V. Candler, D. A. Levin, R. J. Collins, P. W. Erdman, E. Zipf, C. Howlett, Theory of plume radiance from the bow shock ultraviolet 2 rocket flight, Journal of Thermophysics and Heat Transfer 7 (4) (1993) 709-716.

[3] E. Vitkin, V. Karelin, A. Kirillov, A. Suprun, J. V. Khadyka, A physicomathematical model of rocket exhaust plumes, International Journal of Heat and Mass Transfer 40 (5) (1997) 1227-1241.

[4] J. M. Burt, I. D. Boyd, High altitude plume simulations for a solid propellant rocket, AIAA journal 45 (12) (2007) 2872-2884.

[5] P. W. Erdman, E. Zipf, P. Espy, C. Howlett, D. Levin, G. Candler, In-situ measurements of UV and VUV radiation from a rocket plume and re-entry bow shock, AIAA paper (92-0124).

[6] A. Refloch, B. Courbet, A. Murrone, P. Villedieu, C. Laurent, P. Gilbank, J. Troyes, L. Tessé, G. Chaineray, J. Dargaud, et al., Cedre software, AerospaceLab (2) (2011) 1-10.

[7] E. F. Toro, M. Spruce, W. Speares, Restoration of the contact surface in the HLL-Riemann solver, Shock waves 4 (1) (1994) 25-34. 
[8] L. A. Dombrovsky, T. N. Dinh, The effect of thermal radiation on the solidification dynamics of metal oxide melt droplets, Nucl. Eng. Design 238 (6) (2008) 1421-1429.

[9] C. C. Tseng, R. Viskanta, On the hypothesis of thermal phase change, Int. Comm. Heat Mass Transfer 32 (10) (2005) 1267-1272.

[10] S. K. Godunov, A difference method for numerical calculation of discontinuous solutions of the equations of hydrodynamics, Matematicheskii Sbornik 89 (3) (1959) 271-306.

[11] D. J. Carlson, R. F. Hoglund, Particle drag and heat transfer in rocket nozzles, AIAA Journal 2 (11) (1964) 1980-1984.

[12] H. F. Trotter, On the product of semi-groups of operators, Proceedings of the American Mathematical Society 10 (4) (1959) 545-551.

[13] E. Bauer, D. J. Carlson, Mie scattering calculations for micron size alumina and magnesia spheres, Journal of Quantitative Spectroscopy and Radiative Transfer 4 (3) (1964) 363-374.

[14] V. K. Bityukov, V. A. Petrov, Absorption coefficient of molten aluminum oxide in semitransparent spectral range, Applied Physics Research 5 (1) (2013) 51.

[15] R. Reed, V. Calia, Review of aluminum oxide rocket exhaust particles, in: 28th Thermophysics Conference, AIAA, Orlando, Fl, U.S.A, 1993, p. 2819.

[16] L. A. Dombrovsky, Possibility of determining the dispersed composition of a two-phase flow from small-angle light scattering, High Temperature 20 (3) (1982) 472-479.

[17] N. Anfimov, G. Karabadyak, B. Khmelinin, Y. Plastinin, A. Rodionov, Analysis of mechanisms and the nature of radiation from aluminum oxide in different phase states in solid rocket exhaust plumes, in: 28th Thermophysics Conference, AIAA, Orlando, Fl, U.S.A, 1993, p. 2818.

[18] R. M. Goody, Y. L. Yung, Atmospheric radiation: theoretical basis, Oxford University Press, 1995.

[19] J. Taine, A. Soufiani, Gas IR radiative properties: from spectroscopic data to approximate models, Advances in Heat Transfer 33 (1999) 295-414. 
[20] P. Rivière, A. Soufiani, Generalized Malkmus line intensity distribution for $\mathrm{CO} 2$ infrared radiation in Doppler broadening regime, Journal of Quantitative Spectroscopy and Radiative Transfer 112 (3) (2011) 475-485.

[21] L. Soucasse, J. B. Scoggins, Ph. Rivière, T. E. Magin, A. Soufiani, Flowradiation coupling for atmospheric entries using a Hybrid Statistical Narrow Band model, Journal of Quantitative Spectroscopy and Radiative Transfer 180 (2016) 55-69.

[22] C. B. Ludwig, W. Malkmus, J. Reardon, J. Thomson, R. Goulard, Handbook of infrared radiation from combustion gases, Tech. Rep. NASA SP 3080, Washington D.C. (1973).

[23] L. Rothman, I. Gordon, R. Barber, H. Dothe, R. Gamache, A. Goldman, V. Perevalov, S. Tashkun, J. Tennyson, Hitemp, the high-temperature molecular spectroscopic database, Journal of Quantitative Spectroscopy and Radiative Transfer 111 (15) (2010) 2139-2150.

[24] S. Tashkun, V. Perevalov, CDSD-4000: High-resolution, high-temperature carbon dioxide spectroscopic databank, Journal of Quantitative Spectroscopy and Radiative Transfer 112 (9) (2011) 1403-1410.

[25] L. S. Rothman et al, The HITRAN2012 molecular spectroscopic database, Journal of Quantitative Spectroscopy and Radiative Transfer 130 (0) (2013) $4-50$.

[26] W. L. Godson, The computation of infrared transmission by atmospheric water vapor, Journal of Meteorology 12 (3) (1955) 272-284.

[27] S. J. Young, Nonisothermal band model theory, Journal of Quantitative Spectroscopy and Radiative Transfer 18 (1) (1977) 1-28.

[28] J.-M. Lamet, P. Rivière, M.-Y. Perrin, A. Soufiani, Narrow-band model for nonequilibrium air plasma radiation, Journal of Quantitative Spectroscopy and Radiative Transfer 111 (1) (2010) 87 - 104.

[29] L. Tessé, J. Lamet, Radiative transfer modeling developed at onera for numerical simulations of reactive flows, AerospaceLab (2) (2011) 1-19.

[30] H. Smather, D. Horan, J. Cardon, E. Malaret, M. Singh, T. Sorensen, P. Laufer, M. Corson, J. Brandenburg, J. McKay, R. Strunce, Ultraviolet 
Plume Instrument Imaging from the LACE Satellite: The Strypi Rocket Plume, Tech. Rep. NRL/FR/8121-93-9526, Naval Research Laboratory, Washington, D.C. 20375-5320 (September 1993). 